\title{
Flaxseed: a promising alternative for polycystic ovarian syndrome therapy
}

\author{
(D) Sobia Mansoor, @Ayema Haque, @Anooja Rani \\ Dow University of Health Sciences, Department of Internal Medicine, Karachi, Pakistan
}

Cite this article as: Mansoor S, Haque A, Rani A. Flaxseed: a promising alternative for polycystic ovarian syndrome therapy. J Health Sci Med 2021; 4(4): 524-525.

\section{Dear Editor,}

Polycystic ovarian syndrome (PCOS) is a syndrome of ovarian dysfunction associated with menstrual irregularities, hirsutism, obesity, insulin resistance (IR) and hyperinsulinemia. (1) It affects $6-15 \%$ of the female population around the globe (2).

Obesity and IR play a pivotal role in the pathogenesis of PCOS. Obesity causes low-grade inflammation by the production of inflammatory mediators like cytokines, adipokines, and high C-reactive protein (CRP) levels (2). Patients with PCOS are also susceptible to oxidative stress with decreased serum antioxidant levels (2).

For PCOS, lifestyle modification is important which includes weight loss and exercise. A 5\% reduction in weight results in regularity in menstruation and ovulation (2). With the increasing incidence of PCOS, the use of herbal medicines has expanded and have shown promising results in PCOS management which include herbs like Linum usittatissimum (Flaxseed) (4). Flaxseeds(linseeds) is food and fiber that are high in omega 6, 3 fatty acids (alpha linolenic acid, ALA) which have shown benefits in insulin concentration, inflammation as flaxseeds contain Secoisolariciresinol Diglucoside (SDG), which has an anti-inflammatory effect on the human body (5), and oxidative stress by decreasing lipid peroxidation through its antioxidant activity (6). Previous studies (3) show that omega 3 fatty acids supplemented from flaxseed oil when given for 12 weeks improve hyperinsulinemia by reducing the activation of the nuclear factor-kappaB (NF-kB) transcription factor (7) and suppressing proinflammatory mediators, very low density lipoprotein (VLDL)- cholesterol levels and have also shown a reduction in triglyceride levels by increasing lipoprotein lipase activity. All these changes led to an improvement in the manifestations of PCOS. This was also observed in a 2020 randomized controlled trial in which the results showed an improvement in metabolic syndrome and as it is associated with PCOS, flax seeds can be used to treat it (8).

Clinical trials indicate that dietary supplementation with flaxseed in PCOS patients can reduce BMI and weight but had no effect on other anthropometric indices (9). Along with that, a clinical trial demonstrated that flaxseed supplementation shows no side effects, thus, encouraging its use in the management of PCOS (7).

According to a clinical trial, the flaxseed supplementation also leads to decreased leptin levels which is important for the management of obesity (5). Several studies also reported the effect of flaxseed on ovarian morphology and showed a significant reduction in ovarian volume and number of follicles, as well as regularity in menstrual cycles (4).

It is tempting to hypothesize that flaxseeds can decrease the incidence of PCOS by alleviating hypercholesterolemia, hirsutism, insulin resistance, and obesity and the use of flaxseed oil may lead to a reduction in the low-grade inflammation, however, there is confusing literature as some of the studies encourage the use of flaxseed while some of them recommend the use of flaxseed oil to manage PCOS.

Therefore, there is a dire need to design further studies to understand the exact mechanism of action of flaxseeds and their oil in the alleviation of PCOS. Considering the cost-effectiveness, easy availability, minimal side effects, and health benefits, an evidence based purposeful management plan should be developed with flaxseeds and its oil as a part of the medicinal regime in the future..

Keywords: Polycystic ovarian syndrome, flaxseed, flaxseed oil, hyperinsulinemia, oxidative stress. 
Abbreviations: Polycystic ovarian syndrome: PCOS, Insulin resistance: IR, Alpha linolenic acid: ALA, Diabetes Mellitus: DM, Nuclear factor-kappaB: NF-kB, Very low density lipoprotein: VLDL, Secoisolariciresinol Diglucoside: SDG

\section{ETHICAL DECLARATIONS}

Referee Evaluation Process: Externally peer-reviewed.

Conflict of Interest Statement: The authors have no conflicts of interest to declare.

Financial Disclosure: The authors declared that this study has received no financial support.

Author Contributions: All of the authors declare that they have all participated in the design, execution, and analysis of the paper, and that they have approved the final version.

\section{REFERENCES}

1. Swaroop A, Jaipuriar AS, Gupta SK, et al. Efficacy of a novel fenugreek seed extract (Trigonella foenum-graecum, Furocyst $\mathrm{TM}$ ) in polycystic ovary syndrome (PCOS). Int J Med Sci 2015; 12: 825 .

2. Barthelmess EK, Naz RK. Polycystic ovary syndrome: current status and future perspective. Front Biosci (Elite Ed) 2014; 6: 104

3. Pilar B, Güllich A, Oliveira P, Ströher D, Piccoli J, Manfredini V. Protective role of flaxseed oil and flaxseed lignan secoisolariciresinol diglucoside against oxidative stress in rats with metabolic syndrome. J Food Sci 2017; 82: 3029-36.

4. Yadav K, Ghadge P, Langeh A, Kalbhare S, Phadtare P, Bhoite R. A review on herbal medicinal plant for treatment of polycystic ovarian syndrome (PCOS). AJPRD 2020; 8: 83-7.

5. Haidari F, Banaei-Jahromi N, Zakerkish M, Ahmadi K. The effects of flaxseed supplementation on metabolic status in women with polycystic ovary syndrome: a randomized open-labeled controlled clinical trial. Nutr J 2020; 19: 8.

6. Paschos GK, Yiannakouris N, Rallidis LS, et al. Apolipoprotein E genotype in dyslipidemic patients and response of blood lipids and inflammatory markers to alpha-linolenic acid. Angiology 2005; 56: 49-60.

7. Mirmasoumi G, Fazilati M, Foroozanfard F, et al. The effects of flaxseed oil omega-3 fatty acids supplementation on metabolic status of patients with polycystic ovary syndrome: a randomized, double-blind, placebo-controlled trial. Exp Clin Endocrinol Diabetes 2018; 126: 222-8.

8. Yari Z, Cheraghpour M, Hekmatdoost A. Flaxseed and/or hesperidin supplementation in metabolic syndrome: an openlabeled randomized controlled trial. Eur J Nutr. 2021; 60: 287-98.

9. Heidari Z, Ghasemi-Tehrani H, Fallahzadeh H, Nadjarzadeh A. The effects of flaxseed on weight loss in women with polycystic ovarian syndrome: a randomized controlled trial. Iran J Diabetes Obes 2020; 11: 79-86. 